\title{
SV-kNNC: An Algorithm for Improving the Efficiency of k-Nearest Neighbor
}

\author{
Anantaporn Srisawat, Tanasanee Phienthrakul, and Boonserm Kijsirikul \\ Department of Computer Engineering, Faculty of Engineering, Chulalongkorn University, \\ Bangkok 10330 Thailand \\ anantaporn.sestudent.chula.ac.th, tanasaneedyahoo.com, \\ boonserm.k@chula.ac.th
}

\begin{abstract}
This paper proposes SV-kNNC, a new algorithm for k-Nearest Neighbor (kNN). This algorithm consists of three steps. First, Support Vector Machines (SVMs) are applied to select some important training data. Then, $\mathrm{k}$-mean clustering is used to assign the weight to each training instance. Finally, unseen examples are classified by kNN. Fourteen datasets from the UCI repository were used to evaluate the performance of this algorithm. SV-kNNC is compared with conventional $\mathrm{kNN}$ and $\mathrm{kNN}$ with two instance reduction techniques: CNN and ENN. The results show that our algorithm provides the best performance, both predictive accuracy and classification time.
\end{abstract}

\section{Introduction}

In supervised learning, $\mathrm{kNN}$ is one of the most popular choices due to its simplicity [1]. The advantages of kNN include the ability to model complex target functions by a collection of less complex local approximation. Moreover, information presented in the training examples is never lost [2].

However, the main practical difficulties of $\mathrm{kNN}$ are the amounts and characteristics of training data. $\mathrm{kNN}$ methods are based on a distance function for all pairs of a new query instance and training data. If there are a lot of training data, $\mathrm{kNN}$ will take a lot of time in the classification process. In addition, the training data have strong influence on the target output. Noisy data may also reduce the performance of $\mathrm{kNN}$. Hence, if unimportant data and noisy data are eliminated, both classification time and error should be reduced.

This paper proposes SV-kNNC that applies SVM [3] and k-mean clustering [4] to improve the efficiency of $\mathrm{kNN}$. In this approach, SVM are used to reduce the training data of kNN for improving the classification time. Moreover, the accuracy of kNN can also be improved by using k-means clustering to assign weight for each training data.

\section{SV-kNNC}

In this section, we propose SV-kNNC that consists of three processes: instance selection process, weight assigning process, and classification process. The model of SVkNNC is illustrated in Fig. 1. 


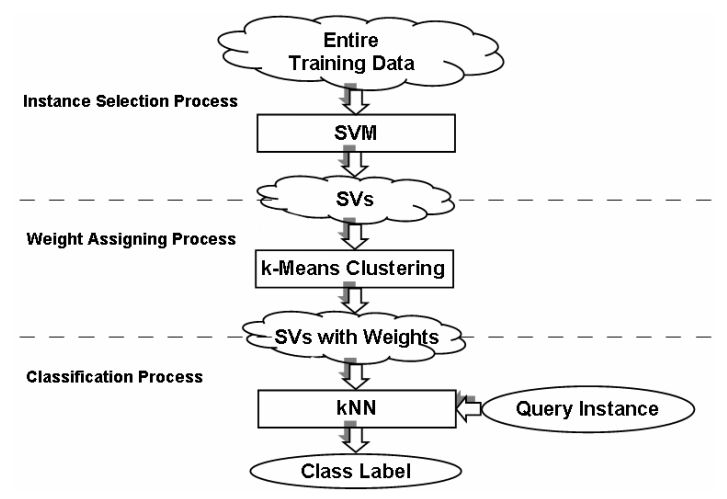

Fig. 1. SV-kNNC Model

\subsection{Instance Selection}

A main difficulty of $\mathrm{kNN}$ is the classification time that increases with the number of training data. If unimportant training data are discarded, the classification time can be reduced. Conventional data reduction methods such as CNN [5], IB3, DROP [6, 7], and ENN [8] gradually add or remove training data. Since these methods do not consider all training data at the same time, they may not yield the actual optimal training set.

We notice that the support vector machine uses only support vectors (SVs), examples that are closest to the hyperplane, to classify unseen data. Therefore, the obtained SVs are representative training data that should be sufficient to represent all training data.

For instance selection process, entire training data are fed into SVM and a set of SVs is produced as the output of SVM training. These SVs are then stored in memory. This process also can eliminate redundant instances of each class in the feature space.

\subsection{Weight Assigning}

While SVs are good representative data for each class in the feature space, some SVs may not be suitable as training data for $\mathrm{kNN}$. This is because the distance between instances is changed when these SVs are retransformed to the input space. The class label of an instance may differ from its surrounding nearest neighbor instances. These instances may be the cause of misclassification.

To avoid this problem, re-checking the contribution of SVs to the classification in the input space before using them to classify a query instance is considered. Weight is then assigned to determine the contribution of each SV. The instance which has a higher weight is more credible, and thus it will have more impact on $\mathrm{kNN}$ classification.

There are 2 steps in weight assigning process. First, the k-means algorithm is used to partition all SVs into pre-defined $k$ clusters. If there are more than one class label appearing in one cluster, the instances with the majority class will be more credible than the instances with the minority classes. After data are clustered, the class labels of all SVs are considered. In this step, each instance in a cluster is assigned weight 
according to the proportion of the instance class labels in that cluster. This weight of sample $x_{i}$, denoted as $w_{i}$, is shown in equation (1).

$$
w_{i}=\frac{n\left(\operatorname{class}\left(x_{i}\right)\right)}{\text { Total }}
$$

where $i=1, \ldots, m$, for $m$ is the number of all SVs. $n\left(\operatorname{class}\left(x_{i}\right)\right)$ is the number of samples in the cluster that have the same class with the sample $x_{i}$. Total is the number of total samples in the cluster.

\subsection{Classification}

In classification process, unseen instances are classified by the $\mathrm{kNN}$ algorithm. Only weighted SVs, which are calculated in the previous process, are used as training data of $\mathrm{kNN}$. When a new query instance is entered, $\mathrm{kNN}$ finds a set of $k$ nearest instances from a set of SVs. Weights of SVs with the same class label are summed up, and the class label with the maximum weight is produced as the class label of the query instance.

\section{Experiments and Results}

In order to verify the performance of our approach, fourteen datasets from the UCI repository [9] are tested using 5-folds crc $\ldots$ validation. Each dataset contains two classes. When SVM, CNN, and ENN were run, the training data were selected differently by these algorithms. Percentages of reduced data are showed in Table 1 . We found that SV-kNNC has the highest ability on data reduction. It reduced data more than CNN and ENN on 6 datasets. Moreover, SV-kNNC reduced training examples up to $71.67 \%$ on the BreastCancer dataset.

Table 1. Percentage of reduced data

\begin{tabular}{lccc}
\hline Datasets & CNN & ENN & SV_kNNC \\
\hline Checkers & 9.11 & 9.38 & $\mathbf{1 0 . 1 6}$ \\
Spiral & 7.65 & 0.00 & $\mathbf{1 4 . 4 3}$ \\
LiverDisorders & 7.32 & $\mathbf{1 7 . 6 1}$ & 14.71 \\
IndiansDiabetes & 10.09 & $\mathbf{1 4 . 7 5}$ & 11.46 \\
ThreeOfNine & $\mathbf{2 9 . 6 4}$ & 10.74 & 0.00 \\
TicTacToe & $\mathbf{3 5 . 4 9}$ & 0.00 & 1.46 \\
BreastCancer & 66.24 & 1.86 & $\mathbf{7 1 . 6 7}$ \\
ParityBits & 8.76 & $\mathbf{1 2 . 0 4}$ & 0.61 \\
ClevelandHeart & 17.69 & 10.00 & $\mathbf{2 4 . 6 3}$ \\
Australian & $\mathbf{2 5 3 6}$ & 0.25 & 15.76 \\
Rand & $\mathbf{1 0 5 8}$ & 0.27 & 2.77 \\
German-org & $\mathbf{1 3 . 8 0}$ & 9.95 & 8.03 \\
Ionosphere & 22.65 & 4.70 & $\mathbf{4 9 . 7 9}$ \\
Sonar & 15.50 & 0.96 & $\mathbf{2 2 . 4 8}$ \\
\hline
\end{tabular}

In Table 2, the accuracies of SV-kNNC are compared with the conventional kNN and kNN with two instance reduction techniques: CNN and ENN. These results show that the accuracies of SV-kNNC are statistically significantly higher than the 
conventional kNN with significance level better than 0.05 on all datasets except for datasets Spiral and ThreeOfNine. The SV-kNNC also provides the best accuracies on all datasets when compared with the other data reduction techniques, i.e. $\mathrm{CNN}$ and ENN.

Table 2. Comparison of accuracies on various algorithms

\begin{tabular}{|c|c|c|c|c|}
\hline Datasets & $\mathrm{kNNN}$ & $\mathrm{CNN}+\mathrm{kNN}$ & $\mathrm{ENN}+\mathrm{kNN}$ & SV-kNNC \\
\hline Checkers & 88.00 & 85.94 & 87.50 & $93.23 \cdots$ \\
\hline Spiral & 100.00 & 98.46 & 100.00 & 100.00 \\
\hline LiverDisorders & 66.09 & 65.51 & 66.67 & $74.20 \cdots$ \\
\hline IndiansDiabetes & 76.82 & 76.95 & 77.21 & $79.03^{* *}$ \\
\hline ThreeOfNine & 100.00 & 96.48 & 92.57 & 100.00 \\
\hline TicTacToe & 97.29 & 93.63 & 97.29 & $99.27^{\cdots *}$ \\
\hline BreastCancer & 97.14 & 96.57 & 96.85 & $98.14^{\circ}$ \\
\hline ParityBits & 53.90 & 56.15 & 49.70 & $68.75^{* *}$ \\
\hline ClevelandHeart & 84.44 & 84.81 & 83.70 & $87.78^{* *}$ \\
\hline Australian & 87.97 & 87.39 & 87.97 & $90.00 \cdots$ \\
\hline Rand & 51.83 & 50.67 & 51.73 & $54.93^{\cdots} \cdots$ \\
\hline German-org & 75.90 & 74.90 & 75.00 & $7690^{\circ}$ \\
\hline Ionosphere & 86.33 & 84.36 & 85.77 & $90.04^{* * *}$ \\
\hline Sonar & 85.56 & 82.68 & 85.09 & $89.87^{* *}$ \\
\hline Average & 82.23 & 81.04 & 81.22 & 85.87 \\
\hline
\end{tabular}

From the experimental results, we found that SV-kNNC enhances the performance of kNN, both classification time and predictive accuracy. Furthermore, the data reduction ability of SV-kNNC is better than CNN and ENN. This is because CNN and ENN keep or remove an instance depending on its nearest instances. Besides, both CNN and ENN are especially sensitive to noise, and noisy instances may be retained. Hence, there are some instances that are still misclassified by CNN and ENN. On the other hand, SVM selects a set of good representative examples of each class by inspecting whole instances at the same time. Thus, some noisy and redundant data may be removed.

\section{Conclusions}

This paper proposes an approach for data reduction to enhance performance of kNN, called SV-kNNC. This algorithm is divided into three steps. First, SVs from SVM learning process are used to be the training examples. Then, these SVs are assigned weights by the ratio of each class label on a cluster from k-mean clustering. Finally, SVs with weights are used to classify the query instances by kNN classification process.

The experimental results showed that SV-kNNC has the ability to reduce data (more than $70 \%$ in some dataset). Thus, the classification time of SV-kNNC is less than kNN. In addition, the accuracy of SV-kNNC is better than the conventional $\mathrm{kNN}$, and $\mathrm{kNN}$ with two instance reduction techniques (CNN and ENN) on the UCI benchmarks.

SV-kNNC achieves the best performance because training data are analyzed twice before classification process. First, SVM eliminates redundant data and selects the instances that are nearest to a decision surface in the feature space. Then, these SVs 
are re-checked and are assigned weights. Therefore, the obtained training data are more effective than the conventional techniques.

\section{Acknowledgement}

The authors acknowledge the financial support provided by the Thailand Research Fund and the Royal Golden Jubilee Ph.D. Program.

\section{References}

1. Cover, T., Hart, P.: Nearest Neighbor Pattern Classification. IEEE Transactions on Information Theory. 13 (1967) 21-27

2. Mitchell, T.M.: Machine Learning. The McGraw-Hill, New York (1997)

3. Vapnik, V.N.: Statistical Learning Theory. John Wiley and Sons, New York (1998)

4. MacQueen, J.B.: Some Methods for classification and Analysis of Multivariate Observations. Proceedings of 5-th Berkeley Symposium on Mathematical Statistics and Probability, Berkeley, University of California Press, 1 (1967) 281-297

5. Hart, P.E.: The Condensed Nearest Neighbor Rule. IEEE Transactions on Information Theory. 14 (1968) 515-516

6. Wilson, D.R., Martinez, T.R.: Reduction Techniques for Instance-Based Learning Algorithms. Machine Learning, 38 (2000) 257-286

7. Jankowski, N., Grochowski, M.: Comparison of Instance Selection Algorithms I. Algorithms Survey. ICAISC (2004) 598-603

8. Wilson, D.L.: Asymptotic Properties of Nearest Neighbor Rules using Edited Data. IEEE Transactions on Systems, Man, and Cybernetics. 2 (1972) 408-421

9. Blake, C.L., Merz, C.J.: UCI Repository of machine learning databases [http:// www.ics.uci.edu/ mlearn/MLRepository.html]. Irvine, CA: University of California, Department of Information and Computer Science (1998) 